\title{
Photocatalytic Degradation of Alizarin Red-S by Fe-Co Nanoparticles Prepared By Chemical Co-precipitation Method
}

\author{
C.V.Nandre ${ }^{1}$ and C.P.Sawant ${ }^{2}$ \\ ${ }^{1}$ North Maharashtra university Jalgaon,Maharashtra. 'Department of Chemistry,Jijamata Education Society's \\ Arts ,Science and College Nandurbar 425412 (M.S.), India. \\ cvnandre@gmail.com \\ ${ }^{2}$ Research laboratory in Chemistry, G.T.Patil College, Nandurbar 425412 (M.S.), India. North Maharashtra \\ university Jalgaon,Maharashtra \\ drcpsawant@rediffmail.com
}

\section{ABSTRACT}

In the present study photocatalytic degradation of hazardous water soluble alizarin red dye by using Fe-Co nanoparticles has been investigated. Fe-Co nanoparticles was synthesized by chemical co-precipitation method and characterized by TEM, SEM, EDAX and XRD. The photocatalytic degradation have been studied with the help of variety of parameters such as catalytic dose, dye concentration, $\mathrm{pH}$, contact time and most important chemical oxygen demand. It was observed that The photocatalytic degradation of alizarin red dye by using $\mathrm{Fe}$-Co nanoparticles was an effective, economic, ecofriendly and faster mode of removing dye from an aqueous solution. The optimum condition for the degradation of the dye was $50 \mathrm{mg} / \mathrm{L}, \mathrm{pH}$ 8.0, catalyst dose $60 \mathrm{mg} / \mathrm{L}$ and contact time 60 minutes. The kinetic studies also have been studied.

Key words: - Chemical co-precipitation; Photocatalytic degradation, Alizarin red dye; Fe-Co nanoparticles; TEM; SEM and XRD.

\section{Council for Innovative Research}

Peer Review Research Publishing System

\section{Journal: Journal of Advances in Chemistry}

Vol. 11, No. 9

www.cirjac.com

editorjaconline@gmail.com, editor@cirjac.com 


\section{INTRODUCTION}

Azo dyes are the largest group of the dyes used for dyeing cotton fabrics in the industry. Cotton is the most widely used fabric among all textile materials, hence azo dyes are discharged frequently and in large quantities into the environment. Due to azo dyes poor exhaustion properties as much as $30 \%$ of the initial dye applied remains unfixed and end up in effluents[1-2]. A necessary criterion for the use these dyes is that they must be highly stable in light and during washing. They must also be resistant to microbial attack. Therefore, they are not readily degradable and are typically not removed from water by conventional chemical wastewater treatment systems. In the past mainly chemical coagulation follows by activated sludge process was adopted to treat textile industrial wastewater. However, azo dyes due to their hydrophilic property are not removed by chemical coagulation. In general, physico-chemical coagulation and flocculation produces large amount of sludge which pose handling and disposal problems. Alizarin red is dark in color which is used for coloring all kind of natural fibers like wool, cotton and silk. It is also used in paints, inks, plastics, leather and cosmetics. However it may cause eye irritation, skin irritation, irritation of digestive tract and may be harmful if swallowed [3-4]. The conventional biological treatment methods are ineffective for the complete color removal and degradation of organics and dyes. The ability of photo catalytic technique to completely degrade organics into $\mathrm{CO}_{2}$ and water without generating any harmful byproducts has popular and its role as wastewater purifier. The technique has been widely employed for the photo mineralization of a large number of dyes such as methylene blue, direct and acid blue dyes, azo dyes and reactive black.6.

During the past decades, the photocatalytic degradation of dyes in water has been promising techniques. Fe-Co nanoparticles are good magnetic material and used as photo catalyst.. Fe-Co nanoparticles are technologically useful material as many dyes were degraded in presence of UV irradiation. Fe-Co nanoparticles are homogeneously dispersed. The focus of the present work is to study the photocatalytic degradation of alizarin red dye by using Fe-Co nanoparticles and UV illumination. There are many methods to fabricate Fe-Co nanoparticles like sol-gel method, chemical bath deposition method and so on [7-8].

In view of the above, we describe our procedure concerning the synthesis of Fe-Co nanoparticles by chemical coprecipitation method and characterization in first part. And the second part contains the photocatalytic degradation of hazardous alizarin red dye.

\section{EXPERIMENTAL DETAILS}

\subsection{Materials and methods}

Fe-Co nanoparticles were synthesized by chemical co-precipitation method. Polyethylene Glycol solution was syringed in to a three neck flask. Then aqueous solutions of $0.05 \mathrm{M}$ Cobalt Nitrate, $0.05 \mathrm{M}$ Ferric Chloride were added with constant stirring. Sodium Borohydride was added slowly drop wise under vigorous stirring, and the stirring was continued for 5 hours. The precipitate obtained was filtered by using Whatman filter paper 41 and precipitates were washed with a deionized water and anhydrous ethanol for several times, and dried in an oven at $150{ }^{\circ} \mathrm{C}$ for $4 \mathrm{~h}$. and milled, and then Fe-Co nanoparticles were obtained [7-9]. For the present investigation water soluble Alizarin red dye, molecular formula $\mathrm{C}_{14} \mathrm{H}_{8} \mathrm{O}_{4}$, molecular weight $(240.21$ $\mathrm{g} / \mathrm{mole}$ ) was selected. $200 \mathrm{mg} / \mathrm{L}$ stock solution of alizarin red was prepared in $1000 \mathrm{ml}$ of distilled water. All chemicals were of analytical grade and used without purification. In $100 \mathrm{ML}$ of dye solution of desired concentration different catalytic dose added and the suspension was irradiated under UV light at different time intervals. The aliquot was taken out with a syringe. Then aliquot was centrifuged and filtered. The concentration of dye solutions were recorded by using UV-VIS. 118 spectrophotometer (Systronic) $\lambda_{\max } 597 \mathrm{~nm}$. All the experiments were carried out at room temperature. The \% degradation of dye has been calculated as:

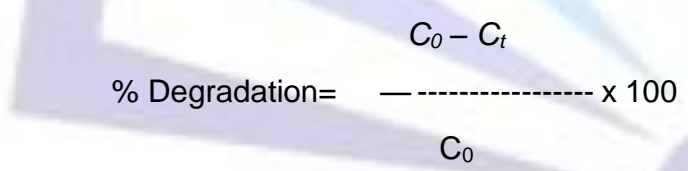

Where $C_{o}$ is the initial concentration of dye and $C_{t}$ is the concentration of dye after photo irradiation[10-12].

\subsection{Instrumentation}

Photo catalytic degradation experiments were carried out in the UV chamber equipped with three UV tubes each of 9 W (Philips) having a wavelength of $365 \mathrm{~nm}$. A magnetic stirrer was used to ensure uniform mixing of solution in vessel. Prior to irradiation the dispersions were magnetically stirred in dark for $15 \mathrm{~min}$. to ensure the establishment of adsorption/desorption equilibrium. The $\mathrm{pH}$ of solutions was measured using digital $\mathrm{pH}$ meter (Systronic) fitted with glass electrode which was previously standardized with buffers of known $\mathrm{pH}$ in acidic and basic medium respectively. For carrying out the COD experiments, reagents were as per standard methods. Usual 2 hrs reflux method was applied for the COD determination. Chemical oxygen demand of colored and photocatalysed solution was determined using standard method. 


\section{RESULTS AND DISCUSSIONS}

\subsection{Effect of catalyst dose}

The effect of photocatalyst concentration on the photodegradation rate of the alizarin red dye was investigated by employing different concentration of Fe-Co nanoparticles varying from 0.1 to $1.0 \mathrm{gL}^{-1}$. As expected the photodegradation rate of the alizarin red was found to increase, then decrease with the increase in the catalyst concentration (fig-1) this phenomenon may be due to the aggregation of Fe-Co particles at high concentration $\left(1.0 \mathrm{gL}^{-1}\right)$ causing a decrease in the

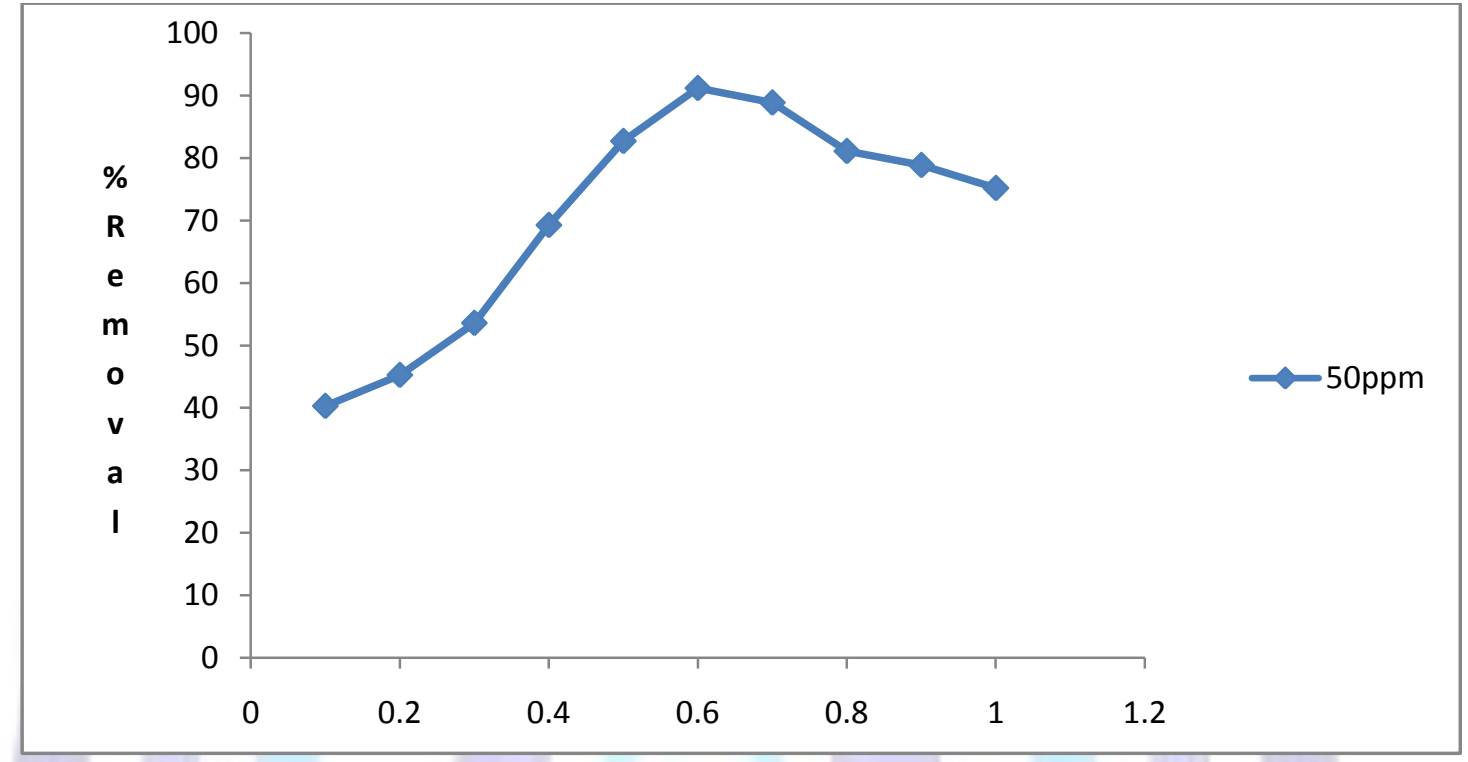

Fig. 1. Effect of catalyst dose on percentage removal of alizarin red by Fe-Co with dye concentration 50ppm with contact time of $60 \mathrm{~min}$. pH 8.0.

number of surface active sites. It is known, however that the scattering light has a practical limit when using high concentration of Fe-Co particles, above which the degradation rate will decrease due to the reduction of the photonic flux within irradiated solution. It was observed that $0.6 \mathrm{gL}^{-1}$ of $\mathrm{Fe}-\mathrm{Co}$ is the optimum dose for efficient degradation of alizarin red dye [13-15].

\subsection{Effect of dye concentration}

In the typical textile effluent, dye concentration ranges from $15 \mathrm{ppm}$ to $30 \mathrm{ppm}$. By varying the initial concentration from $10 \mathrm{ppm}$ to $100 \mathrm{ppm}$ at constant catalyst loading $\left(0.6 \mathrm{gL}^{-1}, \mathrm{pH} 8.0\right)$, its effect on the degradation rate could be determined, and the results are shown in fig. 2. As seen in the figure, degradation efficiency is inversely affected by the dye concentration. This negative effect can be explained as follows; as the dye concentration is increased, the equilibrium adsorption of dye on the catalyst surface active sites increases; hence competitive adsorption of $\mathrm{OH}^{-}$on the same sites decreases, meaning a lower formation rate of ${ }^{*} \mathrm{OH}$ radical, which is the principal oxidant necessary for a high degradation efficiency. On the other hand, considering the Beer-Lambert law, as the initial dye concentration increases, the path length of photons entering the solution decreases, resulting in lower photon adsorption on catalyst particles and, consequently, a lower photodegradation rate [16].

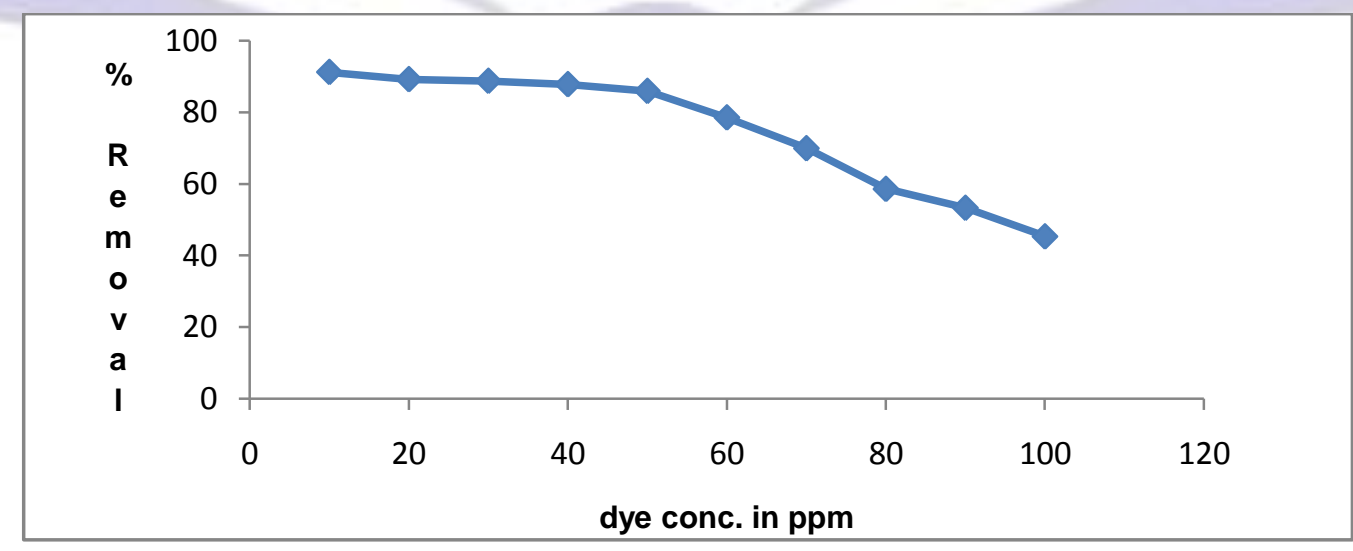

Fig. 2. Effect of initial dye conc., on percentage removal of alizarin red dye by catalyst dose $0.6 \mathrm{gL}^{-1}$ with contact time of 60 min. pH 8. 


\subsection{Effect of $\mathrm{pH}$}

The photocatalytic degradation of dye was studied at different $\mathrm{pH}$ values as it is an important parameter for reaction taking place an particulate surface. The role of $\mathrm{pH}$ on the rate of pho-tocatalytic degradation of alizarin red dye was studied in the $\mathrm{pH}$ range 3.0-12 at dye concentration $50 \mathrm{ppm}$. and catalyst dose $0.6 \mathrm{~g} / \mathrm{L}$. It is apparent that the rate of degradation of alizarin red dye with an increase in the $\mathrm{pH}$ values up to 8.0 and beyond 8.0 the rate of photodegradation becomes constant. This effect may be attributed to more efficient generation of hydroxyl radical by Fe-Co with an increasing concentration of $\mathrm{OH}^{-}$ion upto certain limit. The fact was $\mathrm{pH}$ increases onwards 8.1 the repulsion of the dye anion by negatively charged Fe-Co surface would result in reduction in efficiency of photodegradation of Alizarin red dye[17].

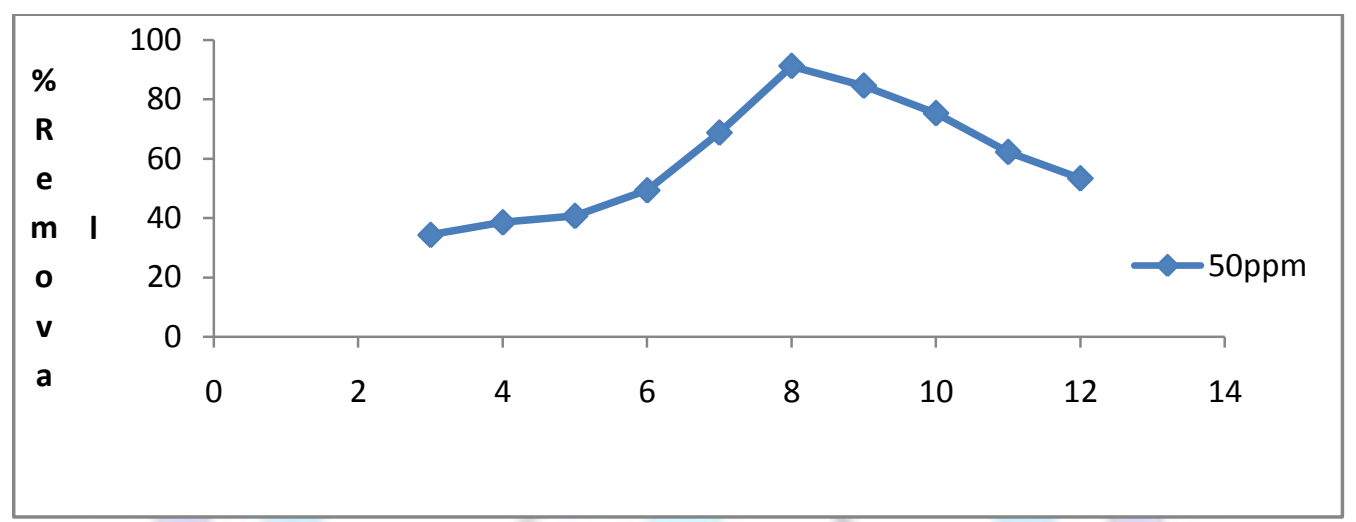

Fig.3. Effect of $\mathrm{pH}$ on percentage removal of alizarin red dye by Fe-Co Catalyst dose $0.6 \mathrm{~g} / \mathrm{L}$ with dye concentration $50 \mathrm{ppm}$ and contact time of $60 \mathrm{~min}$.

\subsection{Effect of contact time}

The effect of contact time for the removal of alizarin red dye by Fe-Co nanoparticles was shown in Figure 4 . The dye was rapidly degraded in the first 60 minutes and then degradation rate decreased gradually and reached equilibrium in about $140 \mathrm{~min}$. At the beginning, degradation rate was fast as the dye ions were adsorbed by the exterior onto the pores of the adsorbent $\mathrm{Fe}-\mathrm{Co}$ and were adsorbed by the interior surface of particle. This phenomenon takes a relatively long contact time[18].

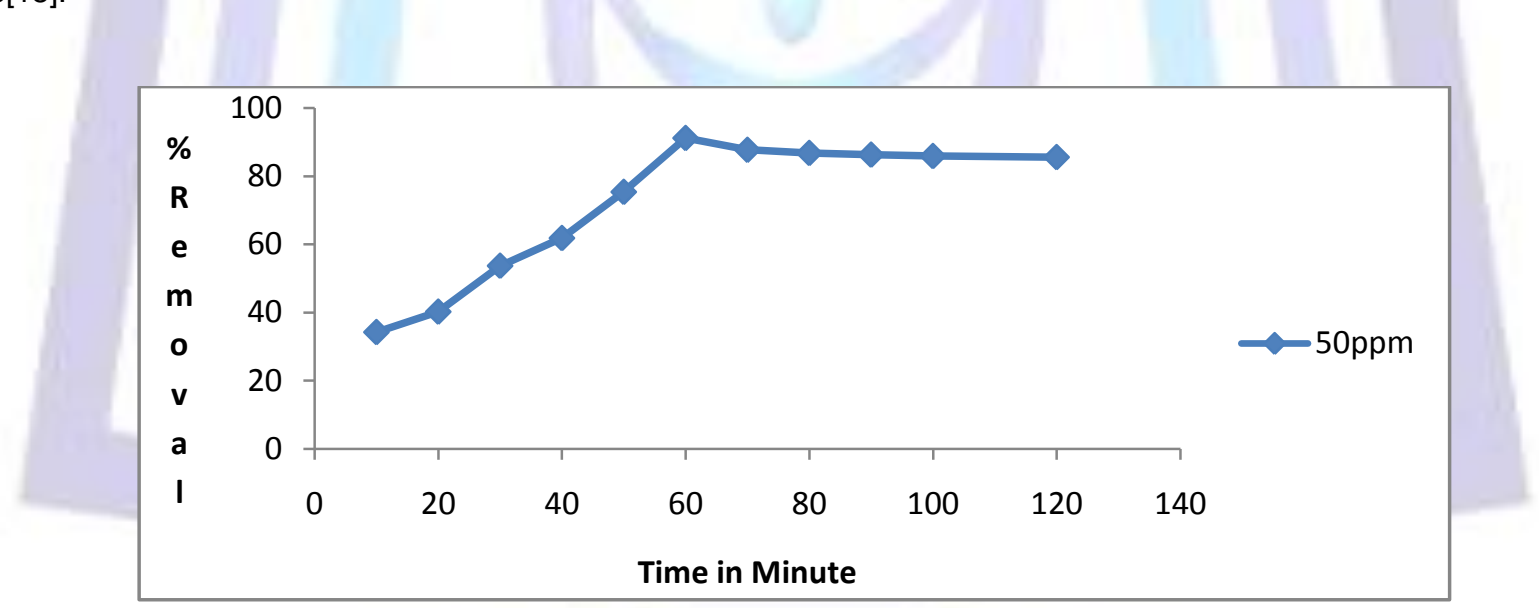

Fig. 4. Effect of contact time on alizarin red dye by catalyst dose $0.6 \mathrm{~g} / \mathrm{L}$ at $\mathrm{pH} 8 . .0$.

\subsection{Effect of electron acceptor}

It is observed that molar $\mathrm{H}_{2} \mathrm{O}_{2}$ concentration is key factor that can significantly influence the degradation of alizarin red dye because $\mathrm{H}_{2} \mathrm{O}_{2}$ concentration is directly related to the no. of $\mathrm{OH}^{-}$radicals generated in the photoassisted reaction. The degradation rate of alizarin red dye increases as the $\mathrm{H}_{2} \mathrm{O}_{2}$ concentration increases until critical $\mathrm{H}_{2} \mathrm{O}_{2}$ concentration is achieved. When using a higher $\mathrm{H}_{2} \mathrm{O}_{2}$ molar concentration, the further generation of $\mathrm{OH}^{-}$radical in aqueous solution is expressed by following Eq. (1).

$$
\mathrm{H}_{2} \mathrm{O}_{2}+\mathrm{OH}^{-} \rightarrow \mathrm{HO}_{2}+\mathrm{H}_{2} \mathrm{O}
$$

Thus, experiments were conducted with different amount of $\mathrm{H}_{2} \mathrm{O}_{2}$ ranging from $1 \mathrm{mg} / \mathrm{L}$ to $10 \mathrm{mg} / \mathrm{L}$ at fixed $\mathrm{pH} 8.0$, dye concentration $50 \mathrm{mg} / \mathrm{L}$ and catalyst dose $60 \mathrm{mg} / \mathrm{L}$. It is apparent that rate of degradation is markedly enhanced in the presence of $\mathrm{H}_{2} \mathrm{O}_{2}$ additive. $\mathrm{H}_{2} \mathrm{O}_{2}$ is not only known to inhibit the electron-hole recombination process, but also generates hydroxyl radicals on abstraction of an electron from the conduction band.

$\mathrm{H}_{2} \mathrm{O}_{2}+\mathrm{e}_{c b}^{-} \rightarrow \mathrm{OH}^{-}+\mathrm{OH}^{-}$ 
It was found that the critical molar $\mathrm{H}_{2} \mathrm{O}_{2}$ concentration for the degradation of alizarin red dye is $4 \mathrm{mg} / \mathrm{L}$, the degradation of efficiency remains constant. This is due to hydroxyl radical scavenging effect of $\mathrm{H}_{2} \mathrm{O}_{2}$.

$$
\begin{gathered}
\mathrm{H}_{2} \mathrm{O}_{2}+\mathrm{OH}^{-} \rightarrow \mathrm{HO}_{2}+\mathrm{H}_{2} \mathrm{O} \\
\mathrm{HO}_{2}+\mathrm{OH}^{-} \rightarrow \mathrm{H}_{2} \mathrm{O}+\mathrm{O}_{2} \quad \text {------.--- (4) }
\end{gathered}
$$

\subsection{Chemical oxygen demand (COD)}

The chemical oxygen demand test is widely used as an effective technique to measure the organic strength of wastewater. The test allow to measurement of waste in terms of total quantity of oxygen required for the oxidation of organic matters to $\mathrm{CO}_{2}$ and $\mathrm{H}_{2} \mathrm{O}$. In the present work results of chemical oxygen demand were taken as one of the parameter to judge the feasibility of photochemical process for the degradation of alizarin red dye solution. In photocatalytic experiments, alizarin red dye $(100 \mathrm{~mL})$ and the catalyst dose $60 \mathrm{mg} / \mathrm{L}$ have taken in a photochemical reactor and exposed to UV light for 60 minutes. Dye sample about 2-3 $\mathrm{mL}$ were taken out at regular interval from the test solution centrifugal at 15-20 minutes and absorbance were recorded at $597 \mathrm{~nm}$. Comparison of the COD value of the initial dye solution with, photocatalysed solution indicates the COD values were substantially reduced. It is observed that the COD values show significant decrease from 1320 to $325 \mathrm{mg} / \mathrm{L}$ indicating good potential of employing photocatalyst technique. The pho-todegradation efficiency was found to be nearby $80 \%$ (Table I)[18-19].

Table I. The COD values before and after dye degradation as shown below.

\section{CHARACTERIZATION}

\subsection{SEM analysis}

\begin{tabular}{|l|l|l|}
\hline Time (min) & $\begin{array}{l}\text { Before treatment } \\
(\mathrm{mg} / \mathrm{L})\end{array}$ & $\begin{array}{l}\text { After treatment } \\
(\mathrm{mg} / \mathrm{L})\end{array}$ \\
\hline 15 & 1320 & 1060 \\
\hline 30 & 995 & 895 \\
\hline 45 & 683 & 584 \\
\hline 60 & 573 & 480 \\
\hline 75 & 405 & 365 \\
\hline 90 & 371 & 325 \\
\hline
\end{tabular}

The SEM images are shown in fig. 5. The observed larger particles exhibited numerous spherical

perturbances on the surface suggesting that they were formed during the precipitation process through fusion of much smaller particles.the prepared products were agglomerated.

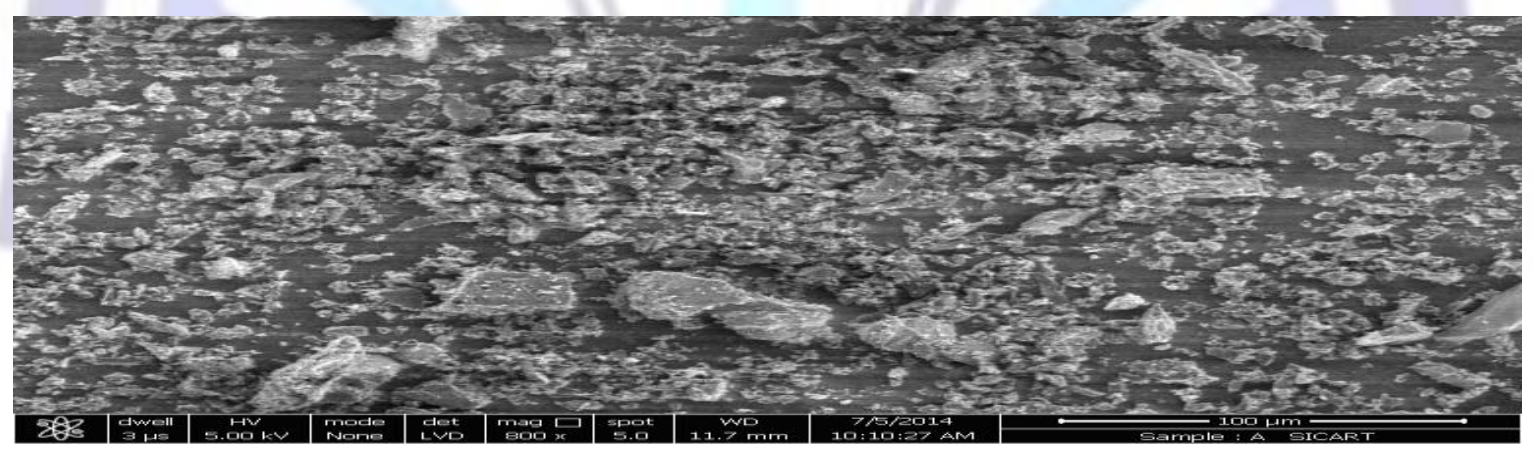

Fig.5 The SEM micrograph of Fe-Co Nanoparticles.

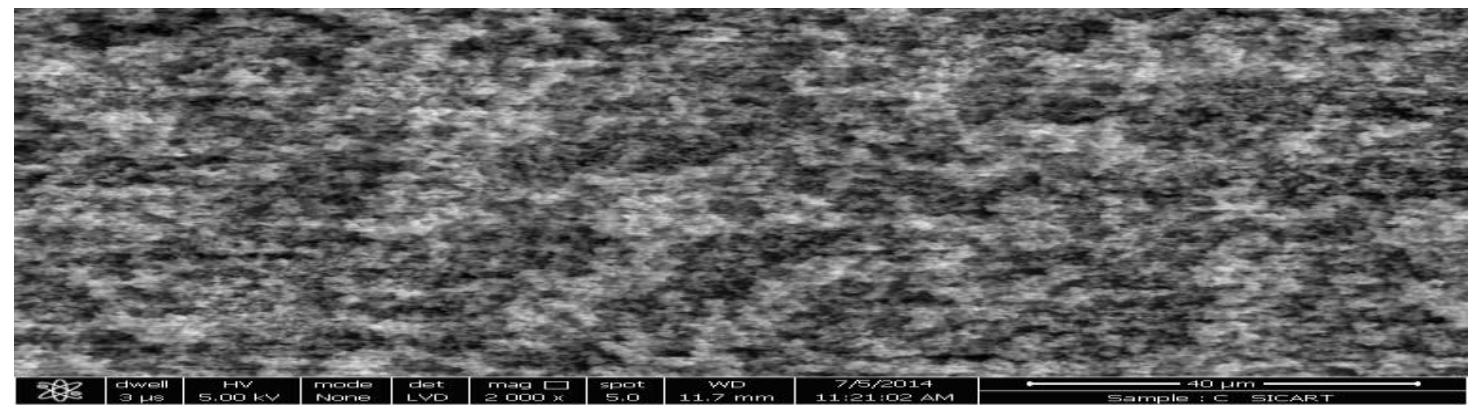

Fig.6 the SEM micrograph of Fe-Co Nanoparticles after photocatalytic degradation. 


\subsection{EDAX analysis}

The EDAX of the Fe-Co nano particles are shown in the fig.7. This provides the conformation of Fe-Co. Apart from Fe and Co carbon and oxygen peaks are also observed in the EDAX spectrum. Presence of carbon protects them from ambient oxidation.

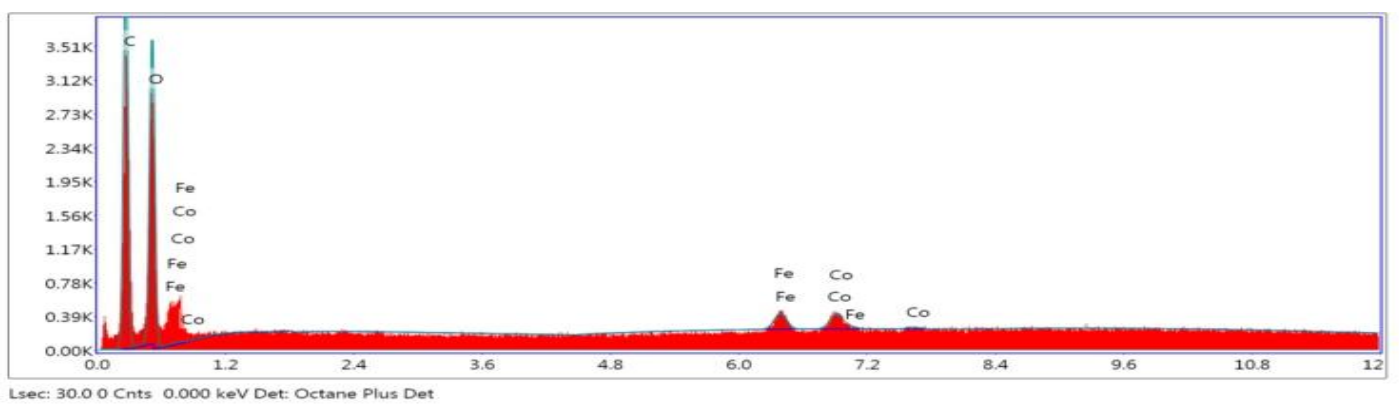

Fig.7. EDAX of the Fe-Co nanoparticles

\subsection{TEM analysis}

Fig.8. shows the TEM images of Fe-Co nanoparticles with good dispertion and size around 20nm. Analysis of the TEM images indicates the nanoparticles are monodisperse with nano size distribution (7\%).

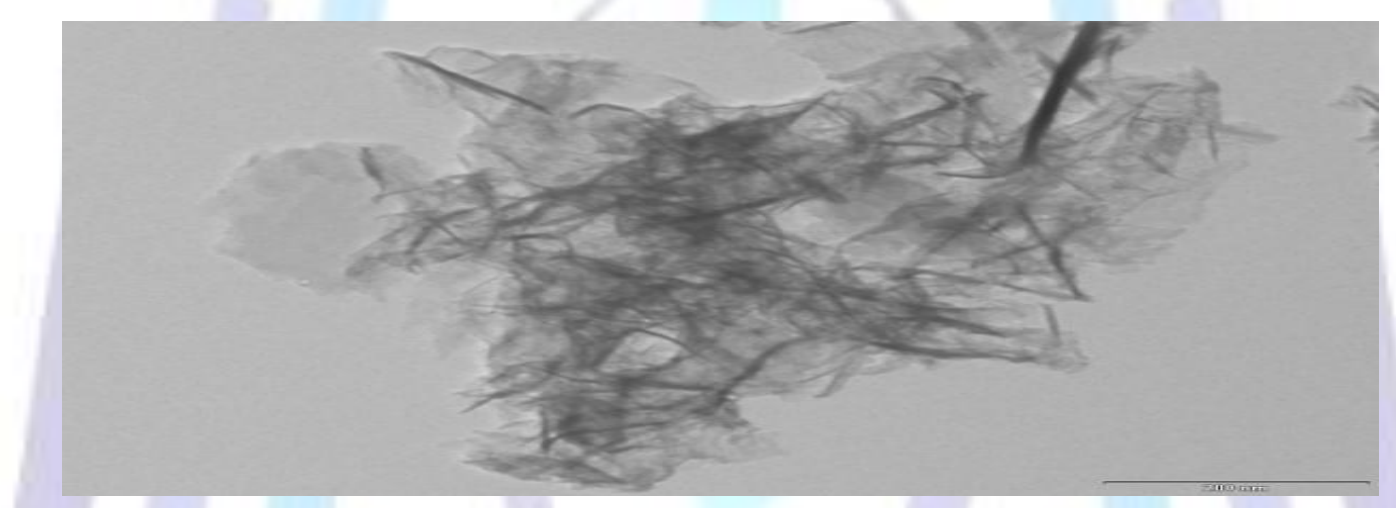

Fig.8. TEM image of Fe-Co nanoparticles

\subsection{XRD analysis}

X-ray diffraction peaks in Fig. 9. are correctly indexed to body centered cubic (bcc) structure of Fe-Co alloy. According to the Debye-Scherrer formula

\section{$\mathrm{D}=\mathrm{K} N / \beta \cos \theta$}

where $\mathrm{K}$ is the Scherrer constant, $\lambda$ the $\mathrm{X}$-ray wavelength, $\beta$ the peak width of half-maximum, and $\theta$ is the Bragg diffraction angle. The average crystallite size $D$ is $20 \mathrm{~nm}$ calculated using the Debye-Scherrer formula, which is very close to the size determined by statistical analysis of TEM images.

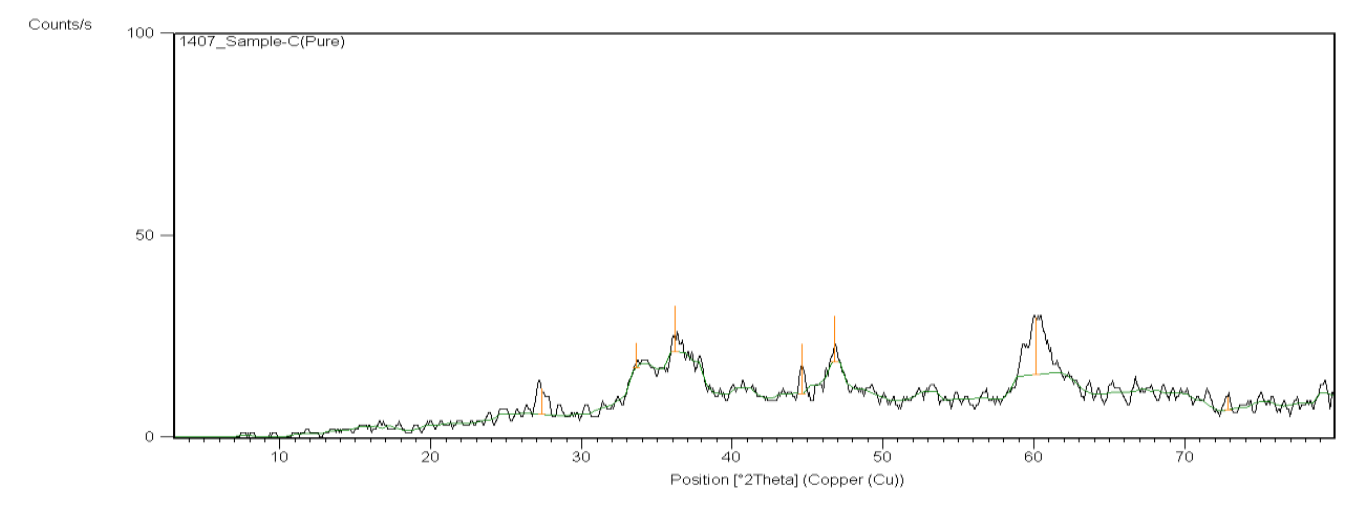

Fig.9. XRD of Fe-Co nanoparticles 


\section{Kinetic Study}

\subsection{Pseudo first order equation}

The pseudo first order equation is generally expressed as

$\log \left(q_{c}-q_{t}\right)=q_{c}-k_{1} / 2.303$

where $q_{e}(\mathrm{mg} / \mathrm{g})$ and $q_{t}(\mathrm{mg} / \mathrm{g})$ are the adsorption capacity at equilibrium and at time $t$ respectively. $k_{1}$ is the rate constant of pseudo first order $\left(\mathrm{min}^{-1}\right)$. The plot of $\log \left(q_{e}-q_{t}\right)$ versus $t$ gives linear relationship from which can be determined from the slope and intercept of the plot respectively (Fig. 10) The linear relationship of the plot for 20 to $50 \mathrm{mg} / \mathrm{L}$. The concentration indicates the validity of equation. The $k_{1}$ values calculated from slope are presented in Table II.

\subsection{Pseudo second order equation}

The pseudo second order equation is expressed as,

$\mathrm{t} / \mathrm{qt}=1 / \mathrm{K} 2 \mathrm{qe}+\mathrm{t} / \mathrm{qe}$ and $\mathrm{h}=\mathrm{K}_{2} \mathrm{qe} \mathrm{e}^{2}$.

Here $\mathrm{K}_{2}$ is rate constant of second order

The plot of $t / q_{t}$ versus $t$ gives linear relationship from which $q_{e}$ and $k_{2}$ can be determined from the slope and intercept respectively. The data showed that good compliance with pseudo first order kinetics model presented in Table II and Figure 11.

Table Il.comparision of the pseudo first order and the pseudo second order adsorption rate constants and calculated and experimental $\mathrm{q}_{\mathrm{e}}$ values for different initial concentrations.

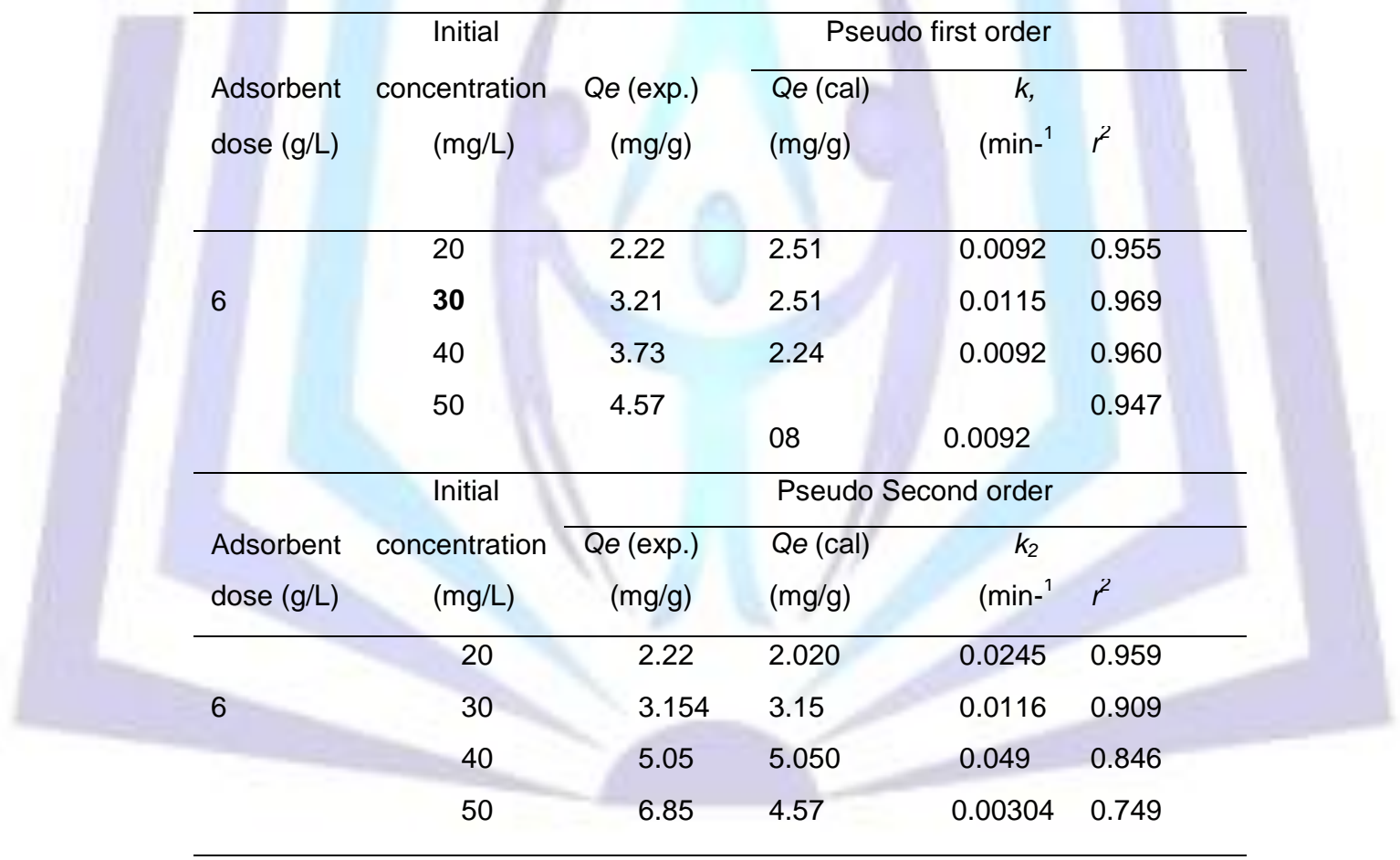

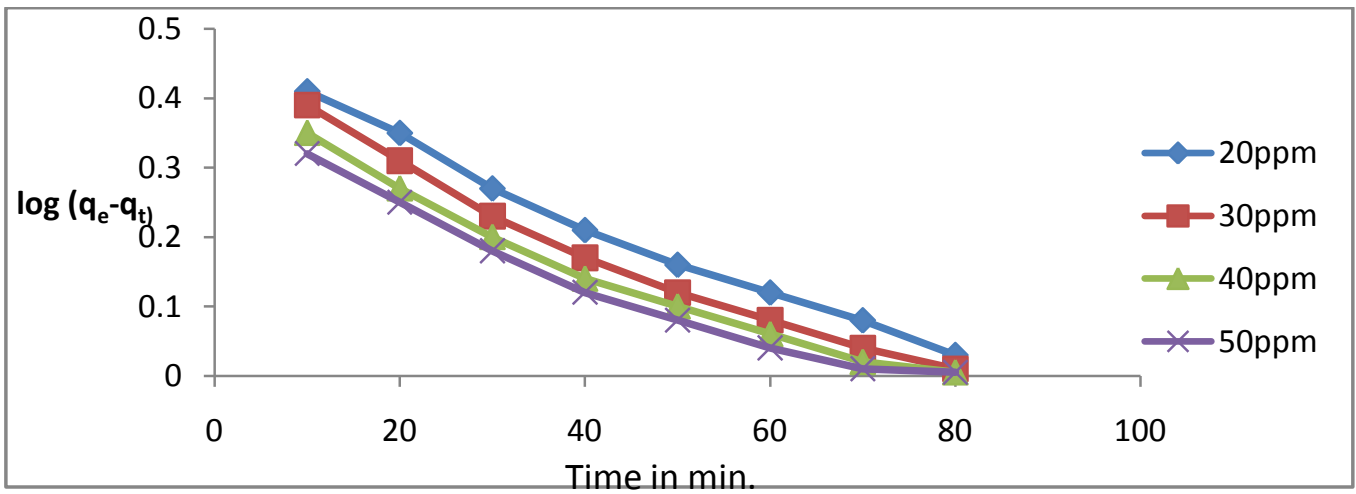

Fig.10. Pseudo $1^{\text {st }}$ order kinetics of alizarin red with catalyst dose $0.6 \mathrm{gm} / \mathrm{L}$ at $\mathrm{pH} 8$. 


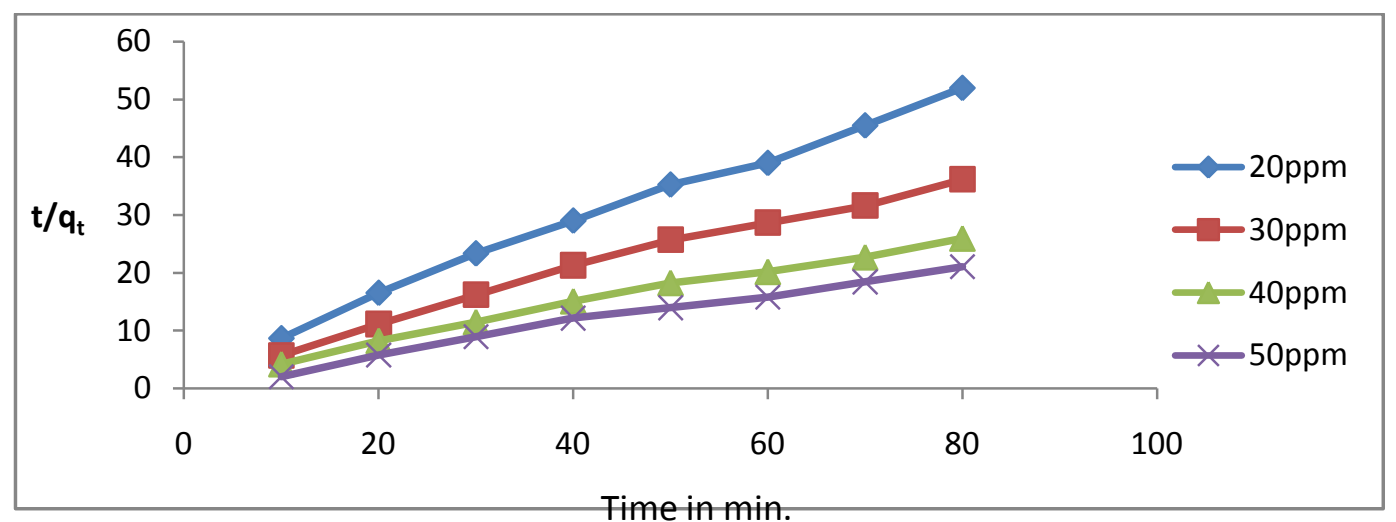

Fig.11. Pseudo 2nd order kinetics of alizarin red with catalyst dose $0.6 \mathrm{gm} / \mathrm{L}$ at $\mathrm{pH} 8$.

\section{CONCLUSIONS}

Photocatalytic degradation using prepared nanosized FRe-Co and UV light was successfully applied for degradation and mineralization of alizarin red dye. The degradation rule was increased significantly by increasing the amount of photocatalyst, while on increasing dye concentration decolorisation deacreases alkaline $\mathrm{pH}$ condition were found to be significantly attend the dye degradation. The XRD study shows utilized Fe-Co was nanosized and porous in nature by SEM. The kinetic study shows that reaction well fitted with pseudo first order model. The maximum degradation efficiency of dye achieved with the concentration of $\mathrm{UV}+\mathrm{H}_{2} \mathrm{O}_{2}+\mathrm{Fe}-\mathrm{Co}$. The photodegradation efficiency of alizarin red dye is $90 \%$.

Acknowledgment: Authors are thankful to the Director, SICART, Vallabh Vidyanagar for XRD,TEM, SEM and EDAX studies. Thanks are also due to Dr. Shirish Sonawane NIT,Varangal for useful discussions. Authors are also thankful to the Principal G.T.P. College, and Principal Jijamata college,Nandurbar for providing necessary laboratory facilities.

\section{RFERENCES}

[1] Bharat,N.Patil., Naik ,D.B. and Shrivastava, V.S.,Sol-gel developed CdS nanoparticles for photocatalytic degradation of alizarin red ,American scientific publisher,4(2011),1-6.

[2] Rajesh.J.Tayade.,Pravin.K.Surolia.,Photocatalytic degradation of dyes and organic contaminants in water using nanocrystalline anatase and rutile $\mathrm{TiO}_{2}$,scince and technology of advanced material,Elsevier, 8(2007), 455-462.

[3] Chen,C.C.,Lu,C.S.,Cang,Y.C.,UV light induced photocatalytic degradation of malachite green on Tio 2 nanoparticles,J.of hazardous material, ,Elsevier, 141(2007) ,520-528.

[4] Ruh,Ullah.,Jaydeep,Dutta., .,Photocatalytic degradation of dyes with manganese doped ZnO nanoparticles, J.of hazardous material, ,Elsevier, 156(2008),194-200.

[5] Joshi,K.M., and Shrivastava,V.S.,Degradation of alizarin red-s by photocatalysis by using $\mathrm{ZnO}$ and $\mathrm{Tio}_{2}$ as photocatalyst,I.J.of environmental science 2(2011),8-21.

[6] Girija,S.Chaube.,Carlos.Barcena.,Narayan.Poudyal.,Synthesis and stabilization of Fe-Co nanoparticles,JACS, 129(2007),7214-7215.

[7] Narayan.Poudyal., Girija,S.Chaube.,Chuan-bing Pong.,Synthesis of monodisperse Fe-Co nanoparticles by reductive salt-matrix annealing,J.of nanotechnology,IOP,24(2013)3456-3458.

[8] Shirsath.S.R.,Hage,A.P.,M.Zhou.,Sonawane, S.H.,M.Ashokkumar.,Ultrasound assisted preparation of nanoclay bentonite- Fe-Co nanocomposite hybrid hydrogel ;a potential responsive sorbent for removal of organic pollutant from water,desalination 281(2011),429-437.

[9] Kim.D.,Yuzh.N.,Kim.B.,Kaidash.E.,Magnetic properties of iron nanoparticles alloyed with cobalt,Glass phys. Chem.33(2007),214-216.

[10] Zainal Z., Hui, L.K., Hussein M. Z., Taufiq-Yap Y. H., Abdullah A. H., and Ramli I., Removal of dyes using immobilized titanium dioxide illuminated by fluorescent lamps. J. Haz. Mat., 125 (2005),113-120.

[11] Banat I.M., Nigam P., Singh D., and Marchant R., Microbial decolorization of textile-dye-containing effluents: a review. Bioresour Technol, 58 (1996) 217-227.

[12] YU Gu.,Yang Cao.,Hujjuan Chi.,Yongil Zhang.and Youvi Sun,Facile synthesis of FeCo/Fe3O4 nanocomposite with high wave absorbing properties.,int.J.of mol sci.14 (2013), 14204-14213.

[13]Soltaninezhad.N.,Aminifar.A.,Study nanostructure of semiconductor zinc oxide as a photocatalyst for organic pollutants, Int. J. of nano dimension2(2011),137-145. 
[14] Muthiralon.C., Nirmala Devi.C.,Minakshi.M.Sundaram.,Synchronous role of coupled adsorption and photocatalytic degradation on CAC-TiO2 composite generating excellent mineralization of alizarin cyanin green dye in aqueous solution.,Arabian J.of chemistry (2013) .

[15] Inam Ullah.,Shakat Ali., Mohmmad Asif Hanif.,Nano science for environmental remediation.,Int.J.of chemical and biological sci.2(2012), 60-77.

[16] Hema,M.,Arivoli,S.,comparative study on the adsorption kinetics and thermodynamics of dyes on to acid activated low cost carbon, International journal of physical sciences,2( 1), (2007),10-17.

[17] Fuat Gnzel, Hasansaygili, Gulbahav, Akkaya Saysilyi, Filizkoyyncy,Decolouration of aqueous crystal violet solution by Nanoporous carbon: Equilibrium and Kinetic approach, Journal of industrial and Engennering Chemistry 1753(2013).

[18] Muftah H. El-Naas, Sulaiman Al-Zuhair, Manal Abu Alhaija, Reduction of COD in refinery wastewater through adsorption on date-pit activated carbon ,Journal of hazardous material, 173 (2010), 750-757.

[19] Pradeep Kumar, B. Prasad, I.M. Mishra, Shri Chand, Decolorisation and COD reduction of dyeing wastewater from a cotton textile mill using thermolysis and coagulation. Journal of Hazardous Materials 153 (2008), 635-645.

[20] Sonawane,G.H.,Shrivastava,V.S.,Kinetics of declourization of malachite green from aqueous medium by maize carbon agricultural solid waste,desalination,250(2009),94-105.

\section{Author biography}

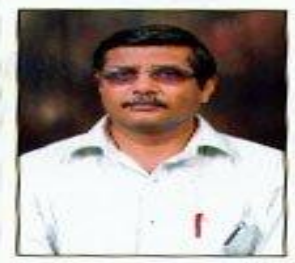

Full name : Nandre Chandrakant Vedu

Birth date: 5.2 .1968

Educational qualification: M.Sc.M.Phil.

Principle subject: Organic Chemistry

Designation: Associate professor

Working place: Dept, of Chemistry, JEEs Arts, Commerce and Science college Nandurbar, Maharashtra (India).

Affiliated university: North Maharashtra University Jalgaon, Maharashtra (India) 425412.

Teaching experience: 23 years

Research experiences: 4 years

Project completed: 1

Research paper published: 03 